\section{Sobrerrastreio mamográfico: avaliação a partir de bases identificadas do Sistema de Informação do Câncer de Mama (SISMAMA)}

\author{
Mammographic over-screening: evaluation based \\ on probabilistic linkage of records databases \\ from the Breast Cancer Information System \\ (SISMAMA)
}

\section{Exceso de pruebas mamográficas: evaluación a partir de las bases identificadas en el Sistema de Información del Cáncer de Mama (SISMAMA)}

COMUNICAÇÃO BREVE

BRIEF COMMUNICATION
Thaís Barbosa Rodrigues 1

Bianca De Stavola 2

Maria Teresa Bustamante-Teixeira 1

Maximiliano Ribeiro Guerra 1

Mário Círio Nogueira 1

Vivian Asssis Fayer 1

Camila Soares Lima Corrêa 1

Isabel dos-Santos-Silva 3

doi: 10.1590/0102-311X00049718

\title{
Resumo
}

No Brasil, o Ministério da Saúde recomenda o rastreamento mamográfico bienal para mulheres entre 50-69 anos. Como o rastreamento é oportunístico no pais, a periodicidade efetiva varia. Esse estudo visou a testar metodologia para a estimação do sobrerrastreio por periodicidade excessiva, definido como intervalo entre exames menores que o preconizado e sua associação com variáveis sociodemográficas. Trata-se de uma coorte de mulheres com mamografia de rastreamento de resultado normal em 2010, obtida por relacionamento probabilistico valendo-se das bases identificadas do SISMAMA. Foram utilizados dados referentes a mulheres residentes na microrregião de saúde de Juiz de Fora/Lima Duarte/Bom Jardim, Minas Gerais, Brasil, acompanhadas no Sistema até o fim de 2012. A taxa de sobrerrastreio foi de 150/mil mulheres/ano (IC95\%: 144,9-155,9), atingindo 21\% das mulheres. O sobrerrastreio aumentou 24\% durante as campanhas Outubro Rosa (HR ajustada = 1,24; IC95\%: 1,15-1,35). Quanto menor o tempo desde a última mamografia, maior foi a chance de sobrerrastreio. Em relação a mulheres que nunca tinham feito mamografia anterior a 2010, as que fizeram há 2 anos foram 2 vezes mais sobrerrastreadas (HR ajustada =2,01; IC95\%: 1,74-2,31) e há $\leq 1$ ano 3 vezes mais (HR ajustada: 3,27; IC95\%: 2,87-3,73). Nessa população, o sobrerrastreio foi substancial, expondo excessivamente as mulheres aos riscos do rastreamento sem benefício adicional e superestimando a cobertura mamográfica. A metodologia mostrou-se efetiva e deve ser aplicada em populações representativas para orientar politicas de controle de câncer de mama.

Programas de Rastreamento; Mamografia; Neoplasias da Mama;

Periodicidade; Pesquisa sobre Serviços de Saúde

\author{
Correspondência \\ T. B. Rodrigues \\ Faculdade de Medicina, Universidade Federal de Juiz de Fora. \\ Rua José Lourenço Kelmer s/n, Juiz de Fora, \\ MG 36036-330, Brasil. \\ tb_rodrigues@yahoo.com \\ 1 Universidade Federal de Juiz de Fora, Juiz de Fora, Brasil. \\ 2 Great Ormond Street Institute of Child Health, University \\ College London, London, U.K. \\ 3 Department of Non-Communicable Diseases Epidemiology, \\ London School of Hygiene and Tropical Medicine, London, U.K.
}




\section{Introdução}

O câncer de mama é o mais incidente 1 , excetuando-se o de pele não-melanoma, e o de maior mortalidade 2 entre as mulheres no Brasil. Estima-se para 2018 a taxa bruta de incidência em 56/100 mil mulheres 1 .

O rastreamento consiste na investigação de indivíduos assintomáticos, e se justifica quando a detecção em estágio pré-clínico e subsequente tratamento da condição em questão proporcionam melhor prognóstico 3 .

O balanço entre benefícios e malefícios dos programas de rastreamento mamográfico organizados é controverso. A redução do risco relativo de morte por câncer de mama após 13 anos foi estimada em 20\% 3. O sobrediagnóstico é considerado o seu grande malefício por acarretar sobretratamento. A chance de mulheres convidadas para tais programas e diagnosticadas com câncer durante o seu curso terem doença que não se manifestaria clinicamente ao longo de suas vidas foi estimada em 19\%, com considerável grau de incerteza 3. Outro malefício é a investigação adicional de falsos positivos. Nessas situações, impõem-se riscos à saúde física e mental, sem ganho individual 3.

O Ministério da Saúde recomenda o rastreamento mamográfico bienal para mulheres entre 50-69 anos, alegando não haver evidência de benefício adicional em maior periodicidade, e ainda maior exposição aos riscos 4 . As recomendações da Organização Mundial da Saúde (OMS) para países com recursos limitados e sistemas de saúde bem estabelecidos são as mesmas. No entanto, enfatiza-se que o balanço final do rastreamento pode ser negativo em programas oportunísticos 5 .

No Brasil, o rastreamento mamográfico é oportunístico, permitindo que a periodicidade se afaste da recomendada 6. O Sistema de Informação do Câncer de Mama (SISMAMA), criado em 2009, armazena dados sobre todas as mamografias realizadas pelo Sistema Único de Saúde (SUS) 7 . Estudos anteriores estimaram indiretamente a periodicidade mamográfica com base no tempo desde o último exame relatado, por meio de inquéritos populacionais 8 ou dados não-individuais do SISMAMA 9. Porém, seus indicadores estão sujeitos a viés de memória.

O objetivo deste estudo piloto é testar metodologia baseada no relacionamento probabilístico de dados identificados do SISMAMA para estimar o sobrerrastreio mamográfico por periodicidade excessiva, assim como sua associação com variáveis sociodemográficas.

\section{Metodologia}

Trata-se de uma coorte de dados administrativos. Foram usados dados identificados do SISMAMA, referentes a mulheres residentes na microrregião de saúde de Juiz de Fora/Lima Duarte/Bom Jardim, Minas Gerais, Brasil. Foram incluídas aquelas com mamografia de rastreio de resultado normal em 2010, definido como Breast Imaging-Reporting and Data System (BI-RADS) 1 ou 2, ou seja, exame negativo ou com achados benignos.

Para evitar superestimação do sobrerrastreio foram excluídas: mulheres que tinham em seu cadastro descrição de nódulo na anamnese, caracterizando mamografia diagnóstica; as de alto risco para câncer de mama, uma vez que a periodicidade de rastreio recomendada é diferente; e as com mamografias alteradas em 2010, realizadas anteriormente à mamografia de rastreamento normal do mesmo ano, já que o menor intervalo poderia ser em consequência do acompanhamento de anormalidades.

O programa RecLink (http://reclink.sourceforge.net/) foi utilizado para construir o banco de dados, incluindo todas as mamografias de cada mulher subsequentes à de resultado normal em 2010, realizadas até o fim de 2012, por meio de relacionamento probabilístico. As variáveis usadas para blocagem foram código soundex do primeiro e último nome e data de nascimento, e para pareamento nome da mãe, município de residência e endereço.

Considerou-se como início do seguimento a data do exame normal de 2010. O desfecho foi sobrerrastreio mamográfico por periodicidade excessiva, definido como mamografia realizada $<18$ meses após resultado normal, com censura administrativa a partir deste momento. Variações de $\leq 6$ meses do preconizado foram consideradas pouco relevantes e possivelmente logísticas.

As variáveis de exposição foram extraídas da solicitação da mamografia normal de 2010. A variável mamografia anterior a 2010 foi construída com base no relato da mulher sobre a data do último 
exame. A variável Outubro Rosa indica se o segundo exame no período de estudo ocorreu entre setembro e novembro.

Utilizou-se para a análise de dados o programa Stata (https://www.stata.com). O percentual de mulheres sobrerrastreadas foi calculado dividindo-se o número de sobrerrastreadas pelo total de mulheres do estudo. A probabilidade cumulativa de fazer nova mamografia de rastreamento após resultado normal foi estimada pelo método de Kaplan-Meier. Para modelar a taxa de sobrerrastreio foram construídos modelos multivariados de Cox, considerando-se o tempo de seguimento como referência temporal. Levando-se em conta não haver benefício em restringir o número de variáveis em um modelo, todas as que não estavam no caminho causal foram incluídas, e então a multicolinearidade foi avaliada usando-se o cálculo do erro médio quadrático. Foram construídos três modelos multivariados. Raça/cor e escolaridade não os compuseram pela extensão de dados ausentes. O pressuposto de proporcionalidade foi avaliado por gráficos de Nelson-Aalen.

Os dados foram fornecidos pela Secretaria de Saúde de Minas Gerais. A presente análise faz parte de pesquisa mais ampla, submetida ao Comitê de Ética em Pesquisa da Universidade Federal de Juiz de Fora (CAE: 46844115.7.0000.5147). Foram tomadas medidas de proteção e de anonimização dos dados.

\section{Resultados}

Foram feitas 19.042 mamografias de rastreio na microrregião em 2010, das quais 16.191 (85\%) apresentaram resultado normal. Após o relacionamento dos dados, verificou-se que advinham de 15.856 mulheres (Figura 1). Após as exclusões, foram incorporadas ao estudo 13.387 mulheres, com 19.031 mulheres/ano de observação.

O sobrerrastreio atingiu 2.860 mulheres (21\%) e sua taxa foi de 150/1.000 mulheres/ano (intervalo de $95 \%$ de confiança - IC95\%: 144,9-155,9). A maioria dos casos de sobrerrastreio $(73,8 \%)$ ocorreu após 1 ano da mamografia normal (Figura 2).

Houve forte evidência de associação de sobrerrastreio com idade, Outubro Rosa e mamografia anterior a 2010 (Tabela 1). Não houve evidência de associação com densidade mamária, raça/cor, escolaridade e exame clínico das mamas após ajuste.

O sobrerrastreio foi maior entre 50-59 e 60-69 anos. Comparado a 50-59 anos, o grupo 40-49 registrou taxa 27\% menor (hazard ratio - HR ajustada = 0,73; IC95\%: 0,67-0,80) e foi o segundo maior em valores absolutos, contendo $28 \%$ das sobrerrastreadas.

Houve aumento de $24 \%$ na taxa de sobrerrastreio durante o período das campanhas Outubro Rosa (HR ajustada = 1,24; IC95\%: 1,15-1,35).

As mulheres que fizeram mamografia antes de 2010 exibiram taxas de sobrerrastreio maiores do que aquelas cuja primeira mamografia da vida foi efetuada neste ano, sendo as taxas progressivamente maiores com o menor tempo desde a realização da última mamografia, a saber: há $\geq 3$ anos apresentaram taxa 27\% maior (HR ajustada = 1,27; IC95\%: 1,07-1,51), há 2 anos 2 vezes maior (HR ajustada = 2,01; IC95\%: 1,74-2,31) e há $\leq 1$ ano 3 vezes maior (HR ajustada = 3,27; IC95\%: 2,87-3,73).

\section{Discussão}

Demonstrou-se nesta população sobrerrastreio substancial, afetando $21 \%$ das mulheres, e expondo-as mais aos malefícios advindos do rastreamento, sem maiores ganhos em redução de mortalidade 3,4,5. Tais resultados têm impacto nas estimativas de cobertura mamográfica que, em geral, são calculadas com número de exames/população alvo 9, levando a superestimações. Adicionalmente, os resultados demonstram haver ônus desnecessário para o SUS.

O acréscimo de $24 \%$ no sobrerrastreio durante o Outubro Rosa pode ser creditado ao aumento da disponibilidade de serviços ou enfoque excessivo de tais campanhas no rastreamento, em detrimento da conscientização sobre a doença. O aumento importante no sobrerrastreio com menor tempo desde a última mamografia relatada pode refletir acesso diferenciado de parcela das mulheres à informação e serviços de saúde. 
Figura 1

Etapas de elaboração do banco de dados.

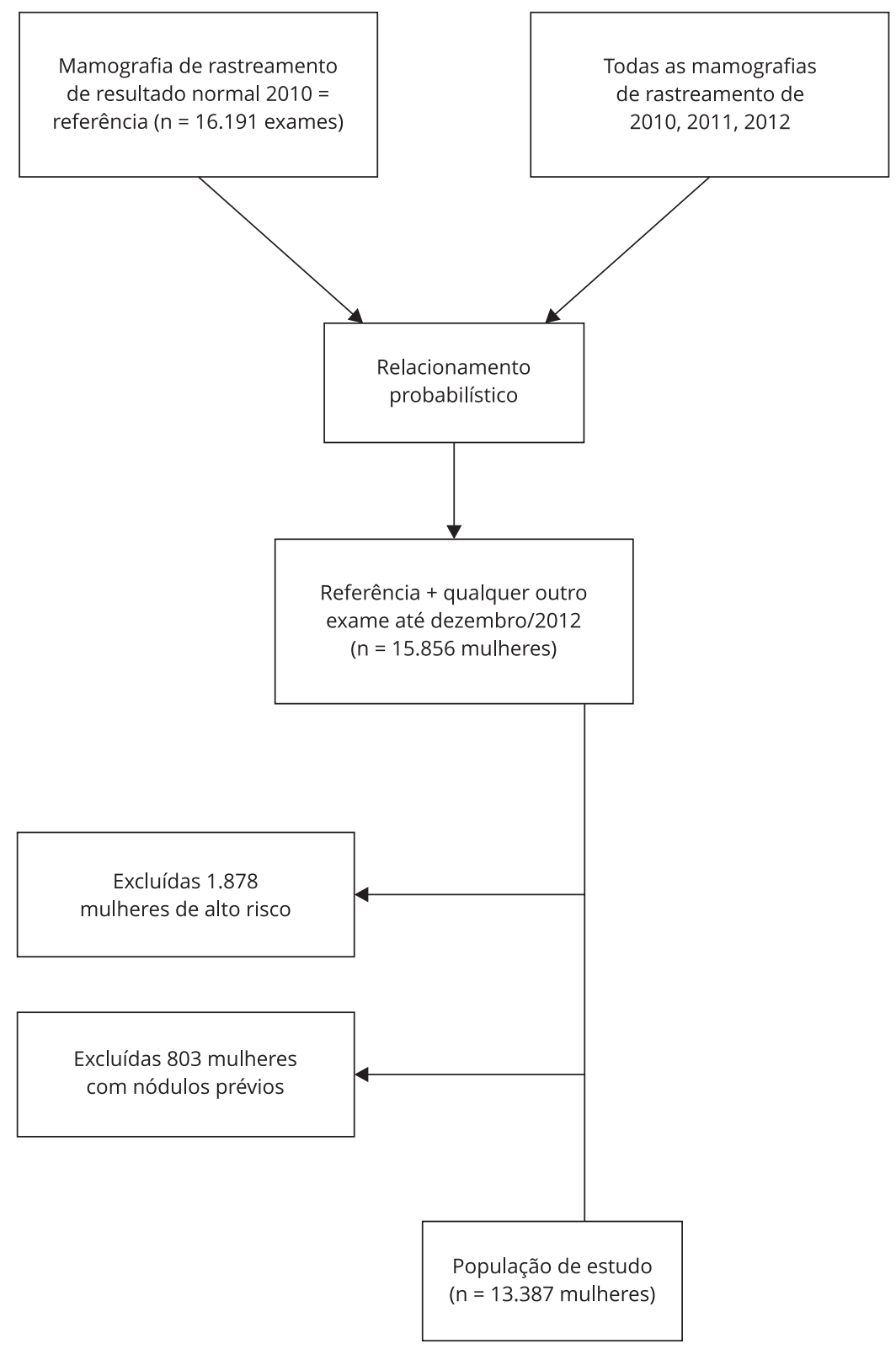

Erros no relacionamento probabilístico poderiam alterar a estimativa de sobrerrastreio, porém, são improváveis pela sua alta acurácia 10 e seriam não diferenciais. Poderia haver subestimação do sobrerrastreio se mulheres fizessem mamografia subsequente na rede privada ou em outra microrregião, porém não há motivo a priori para supor que isto ocorra frequentemente.

O sobrerrastreio pode ter sido superestimado caso mamografias diagnósticas que compõem o protocolo de acompanhamento de BI-RADS 3 fossem erroneamente cadastradas como de rastreamento. As exclusões feitas visaram a reduzir tal possibilidade. A frequência de BI-RADS 3 em mamografias de rastreamento em Minas Gerais foi estimada em 3,3\% em 2011 9, logo, tal fenômeno só afetaria parcialmente o desfecho. 


\section{Figura 2}

Kaplan-Meier do percentual cumulativo de mulheres sobrerrastreadas em seguida à mamografia de rastreio de resultado normal $(n=2.860)$.

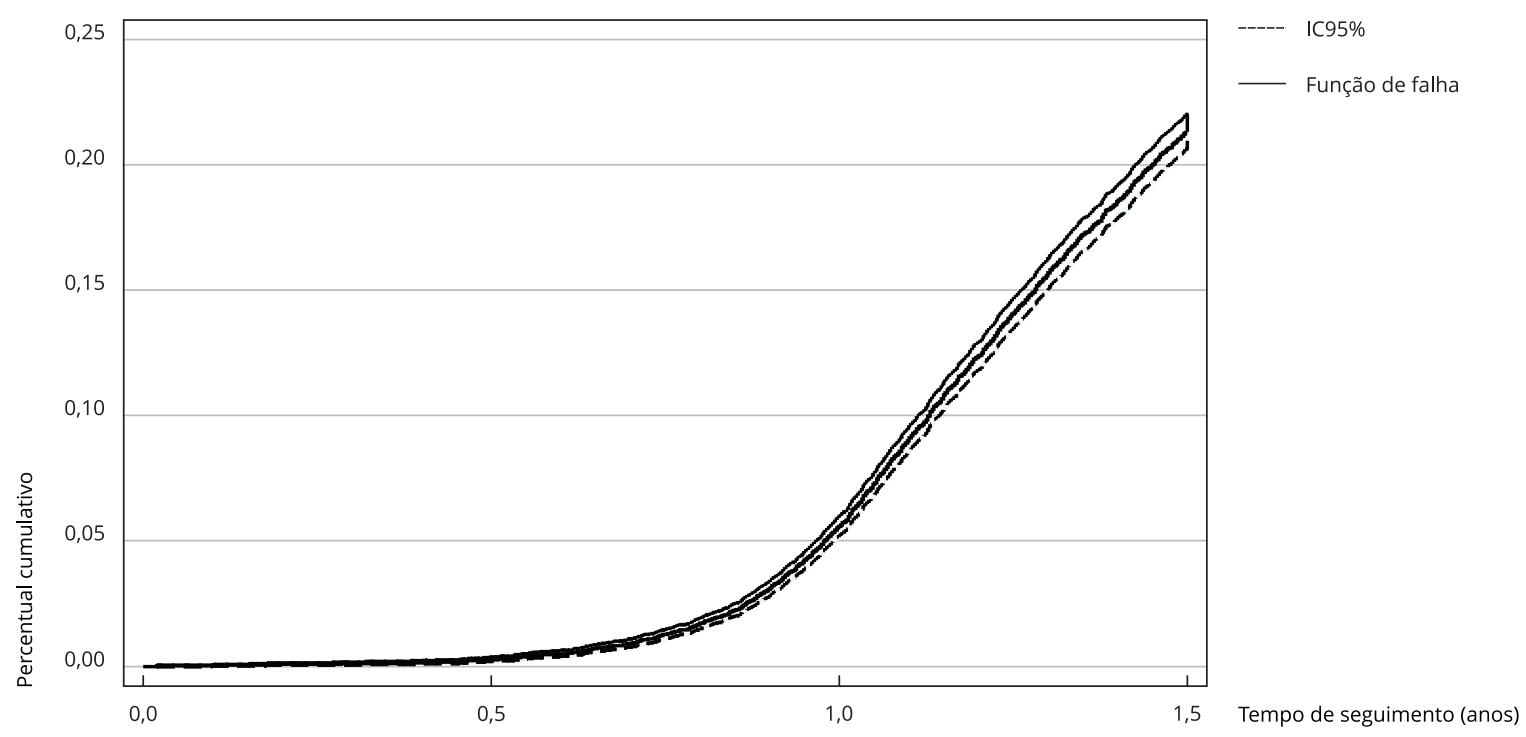

IC95\%: intervalo de 95\% de confiança.

Nota: tempo de seguimento (anos) - mulheres sob risco: 0-13.387; 0,5-13.352; 1,0-12.639; 1,5-0.

O uso exclusivo de dados administrativos representa uma relevante limitação, já que informações sobre exposições são coletadas sem padronização e sujeitas a erros. Porém, erros seriam não diferenciais e enviesariam as medidas em direção a um efeito nulo. Além disso, limitou a avaliação de indicadores socioeconômicos. Raça/cor e escolaridade registraram grande porcentual de dados ausentes, não imputados por não serem necessariamente aleatórios.

Estimativas de sobrerrastreio de estudos anteriores não são diretamente comparáveis às deste devido a diferentes definições de desfecho e populações, a não exclusão de alto risco e especialmente por dependerem de relatos, sujeitos ao viés de memória.

Por tratar-se de estudo piloto, os resultados não são diretamente aplicáveis a outras regiões. No entanto, a metodologia empregada mostrou-se efetiva em estimar o sobrerrastreio e deve ser aplicada futuramente utilizando dados estaduais ou macrorregionais, de forma a orientar políticas de controle de câncer de mama baseadas em evidências no Brasil. 
Tabela 1

Possíveis associações com sobrerrastreio mamográfico em uma coorte de 13.387 mulheres (eventos: 2.860 mulheres sobrerrastreadas; acompanhamento: 19.031 mulheres/ano).

\begin{tabular}{|c|c|c|c|c|c|c|}
\hline & Eventos & Mulheres/Ano & $\begin{array}{c}\text { HR bruta * } \\
\text { (IC95\%) }\end{array}$ & Valor de $p * *$ & HR ajustada * (IC95\%) & Valor de $p$ ** \\
\hline Idade atual (anos) $\star * \star, \#, \# \#$ & & & & $<0,001$ & & $<0,001$ \\
\hline$<40$ & 24 & 642 & $0,23(0,15-0,34)$ & & $0,22(0,15-0,34)$ & \\
\hline $40-49$ & 802 & 6.453 & $0,73(0,67-0,80)$ & & $0,73(0,67-0,80)$ & \\
\hline 50-59 (Ref.) & 1.221 & 6.954 & 1,00 & & 1,00 & \\
\hline $60-69$ & 646 & 3.498 & $1,03(0,93-1,13)$ & & $1,03(0,93-1,13)$ & \\
\hline$\geq 70$ & 167 & 1.465 & $0,60(0,51-0,71)$ & & $0,60(0,51-0,71)$ & \\
\hline Outubro Rosa \#\#\# & & & & $<0,001$ & & $<0,001$ \\
\hline Não (Ref.) & 1.979 & 14.145 & 1,00 & & 1,00 & \\
\hline Sim & 881 & 4.886 & $1,27(1,17-1,37)$ & & $1,24(1,15-1,35)$ & \\
\hline Raça/Cor § & & & & 0,63 & & - \\
\hline Branca (Ref.) & 177 & 1.201 & 1,00 & & - & \\
\hline Outras & 52 & 385 & $0,93(0,68-1,26)$ & & - & \\
\hline Escolaridade §§ & & & & 0,80 & & - \\
\hline Ensino Médio completo (Ref.) & 83 & 581 & 1,00 & & - & \\
\hline Não & 431 & 2.910 & $1,03(0,81-1,30)$ & & - & \\
\hline Mamografia anterior a 2010 \#\#\# & & & & $<0,001$ & & $<0,001$ \\
\hline Nenhuma (Ref.) & 296 & 4.120 & 1,00 & & 1,00 & \\
\hline Não sabe & 334 & 2.645 & $1,81(1,55-2,12)$ & & $1,73(1,48-2,03)$ & \\
\hline$\geq 3$ anos & 260 & 2.629 & $1,40(1,18-1,65)$ & & $1,27(1,07-1,51)$ & \\
\hline 2 anos & 645 & 4.172 & $2,23(1,95-2,56)$ & & $2,01(1,74-2,31)$ & \\
\hline$\leq 1$ ano & 1.325 & 5.466 & $3,67(3,24-4,16)$ & & $3,27(2,87-3,73)$ & \\
\hline Densidade da mama esquerda §§§ & & & & 0,91 & & 0,49 \\
\hline Densa (Ref.) & 451 & 2.973 & 1,00 & & 1,00 & \\
\hline Predominantemente densa & 1.285 & 8.445 & $1,00(0,90-1,12)$ & & $1,00(0,90-1,12)$ & \\
\hline Predominantemente adiposa & 1.025 & 6.927 & $0,98(0,88-1,09)$ & & $1,06(0,95-1,19)$ & \\
\hline Adiposa & 99 & 686 & $0,96(0,77-1,19)$ & & $1,12(0,90-1,40)$ & \\
\hline Exame clínico das mamas \#\#\# & & & & $<0,001$ & & 0,18 \\
\hline Nunca (Ref.) & 118 & 1.250 & 1,00 & & 1,00 & \\
\hline Sim & 2.742 & 17.782 & $1,67(1,39-2,01)$ & & $1,14(0,94-1,38)$ & \\
\hline
\end{tabular}

HR: hazard ratio; IC95\%: intervalo de 95\% de confiança; Ref.: referência.

* Modelo de Cox com seguimento como escala de tempo;

** Likelihood ratio test para associação com sobrerrastreio;

*** Faltando 13 pessoas e 19 anos/pessoa;

\# HR ajustada: para Outubro Rosa;

\#\# Idade atual modelada por meio de lexis expansion;

\#\# HR ajustada: para todas as demais variáveis;

§ Faltando 12.271 pessoas e 17.445 mulheres/ano;

§§ Faltando 10.934 pessoas e 15.540 mulheres/ano;

$\S \S \S$ HR ajustada: idade e Outubro Rosa. 


\section{Colaboradores}

T. B. Rodrigues contribuiu na concepção do projeto, análise dos dados e redação do artigo. B. De Stavola colaborou na concepção do projeto, preparo e análise dos dados, orientação para as análises e revisão crítica do conteúdo. M. T. Bustamante-Teixeira, M. R. Guerra, M. C. Nogueira, V. A. Fayer e C. S. L. Corrêa contribuíram na concepção do projeto, relacionamento probabilístico dos dados, revisão crítica do conteúdo. I. dos-Santos-Silva colaborou na ideia original, concepção do projeto, orientação para as análises e revisão crítica do conteúdo.

\section{Agradecimentos}

Os autores agradecem às fontes de financiamento deste estudo: Graduação Sanduíche do Programa Ciência sem Fronteira - CNPq (Brasil); e Fundo Newton - Research Councils (Reino Unido) e CONFAP (Brasil), como parte do projeto Developing Tools to Study Inequalities in Women's Access to Breast and Cervical Cancer Control Activities in Brazil Using Health-Related Databases.

\section{Referências}

1. Coordenação de Prevenção e Vigilância, Instituto Nacional de Câncer José Alencar Gomes da Silva. Estimativa 2018: incidência de câncer no Brasil. Rio de Janeiro: Instituto Nacional de Câncer José Alencar Gomes da Silva; 2017.

2. Instituto Nacional de Câncer José Alencar Gomes da Silva. Atlas on-line de mortalidade. https://mortalidade.inca.gov.br/Mortalidade $\mathrm{Web} /$ (acessado em 12/Fev/2018).

3. Marmot MG, Altman DG, Cameron DA, Dewar JA, Thompson SG, Wilcox M, et al. The benefits and harms of breast cancer screening: an independent review. Br J Cancer 2013; 108:2205-40.

4. Instituto Nacional de Câncer José Alencar Gomes da Silva. Diretrizes para a detecção precoce do câncer de mama no Brasil. Rio de Janeiro: Instituto Nacional de Câncer José Alencar Gomes da Silva; 2015.

5. World Health Organization. WHO position paper on mammography screening. Geneva: World Health Organization; 2014.

6. Migowski A, Dias MBK, Nadanovsky P, Azevedo e Silva G, Sant'Ana DR, Stein AT. Diretrizes para detecção precoce do câncer de mama no Brasil. III - Desafios à implementação. Cad Saúde Pública 2018; 34:e00046317.

7. Passman LJ, Farias AMRO, Tomazelli JG, Abreu DMF, Dias MBK, Assis M, et al. SISMAMA implementation of an information system for breast cancer early detection programs in Brazil. Breast 2011; 20 Suppl 2:S35-9.

8. Lima-Costa MF, Matos DL. Prevalência e fatores associados à realização da mamografia na faixa etária de 50-69 anos: um estudo baseado na Pesquisa Nacional por Amostra de Domicílios (2003). Cad Saúde Pública 2007; 23:1665-73.

9. Corrêa CS, Pereira LC, Leite ICG, Fayer VA, Guerra MR, Bustamante-Teixeira MT. Rastreamento do câncer de mama em Minas Gerais: avaliação a partir de dados dos sistemas de informações do Sistema Único de Saúde. Epidemiol Serv Saúde 2017; 26:481-92.

10. Camargo Jr. KR, Coeli CM. RecLink 3: nova versão do programa que implementa a técnica de associação probabilística de registros (probabilistic record linkage). Cad Saúde Colet (Rio J.) 2006; 14:399-404. 


\section{Abstract}

The Brazilian Ministry of Health recommends biennial mammographic screening for women aged between 50 and 69 years. Since screening is opportunistic in the country, the actual periodicity varies. This study sought to test a methodology for estimating over-screening due to excessive periodicity, defined as a smaller than recommended interval between exams, and its association with socio-demographic characteristics. A cohort of women who underwent mammography in 2010, and whose result was normal, was assembled through probabilistic linkage SISMAMA records based on a set of personal identifiers. We used data from women living in the micro health region of Juiz de Fora/Lima Duarte/Bom Jardim, Minas Gerais State, Brazil, who were followed in the System until the end of 2012. The rate of over-screening was 150/1,000 women/year (95\%CI: 144.9-155.9), affecting $21 \%$ of women. Over-screening increased by 24\% during Pink October campaigns (adjusted $H R=1.24$; 95\%CI: 1.15-1.35). The shorter the time passed since the last mammogram, the greater the odds of over-screening. Compared with women who had never had a mammogram prior to 2010, women who had had one in the previous 2 years were two times more likely to be overscreened (adjusted HR = 2.01; 95\%CI: 1.74-2.31) whilst those who had had a mammogram $\leq 1$ year previously were three times more likely to be overscreened (adjusted HR = 3.27; 95\%CI: 2.87-3.73). Over-screening was substantial in this population, excessively exposing women to the risks of screening with no additional benefits and overestimating mammogram coverage. The methodology proved to be successful and should be applied to representative populations in order to guide breast cancer control policies.

Mass Screening; Mammography;

Breast Neoplasms; Periodicity;

Health Services Research

\section{Resumen}

En Brasil, el Ministerio de Salud recomienda pruebas mamográficas bienales para mujeres entre 50-69 años. Como las pruebas se realizan ocasionalmente en el país, la periodicidad efectiva varía. El objetivo de este estudio fue probar la metodología para la estimación del exceso de pruebas por periodicidad excesiva, definido como un intervalo menor entre exámenes que el preconizado, y su asociación con variables sociodemográficas. Se trata de una cohorte de mujeres con mamografías para la detección de cáncer con un resultado normal en 2010, obtenida mediante relación probabilística, haciendo uso de las bases identificadas del SISMAMA. Se utilizaron datos referentes a mujeres, residentes en la microrregión de salud de Juiz de Fora/Lima Duarte/Bom Jardim, Estado de Minas Gerais, Brasil, a quienes se les realizó un seguimiento en el sistema hasta finales de 2012. La tasa de exceso pruebas fue de 150/1.000 mujeres/ año (IC95\%: 144,9-155,9), alcanzando un 21\% de las mujeres. El exceso de pruebas aumento un $24 \%$ durante las campañas Octubre Rosa (HR ajusta$d a=1,24$; IC95\%: 1,15-1,35). Cuanto menor era el tiempo desde la última mamografía, mayor fue la oportunidad de exceso de pruebas. En relación con mujeres que nunca se habian hecho una mamografía anterior a 2010, en quienes se la hicieron hace 2 años hubo 2 veces más exceso de pruebas (HR ajustada $=2,01$; IC95\%: 1,74-2,31) y hace $\leq$ 1 año 3 veces más (HR ajustada = 3,27; IC95\%: 2,87-3,73). En esta población, el exceso de pruebas fue sustancial, exponiendo excesivamente a las mujeres a los riesgos de la detección sin beneficio adicional y sobrevalorando la cobertura mamográfica. La metodología se mostró efectiva y se debe aplicar en poblaciones representativas para orientar políticas de control de cáncer de mama.

Tamizaje Masivo; Mamografía; Neoplasias de la Mama; Periodicidad; Investigación en Servicios de Salud
Recebido em 12/Mar/2018

Versão final reapresentada em 16/Ago/2018 Aprovado em 09/Nov/2018 